\title{
General Self-energy based Formulation of Levels Coupling in Quantum Confined Structures
}

\author{
Marco Vallone ${ }^{1}$ \\ Dipartimento di Elettronica, Politecnico di Torino, Torino 10126, Italy \\ PACS: $\quad$ 85.35.Be $\quad$ 73.21.Fg $\quad$ 42.55.Px $\quad$ 71.70.-d
}

The effect of coupling between levels in quantum wells or quantum dots is described in the Green's function formalism. The structure eigenvalues are shown to have a Brillouin-Wigner continued fraction expression that allows to give a general and intuitive meaning to levels-coupling, described in terms of an off-diagonal self-energy. The concept of coupling is linked to a general potential matrix and can be given the same mathematical form for all kind of coupling (inter-and intra-quantum dot and quantum well), in which off-diagonal self-energy contributions assume each time a different conceptual meaning. Furthermore, the same scheme, based on off-diagonal self-energies, allows to evaluate renormalization contribution due to each structure energy level in a natural and easy way.

Index Terms - Quantum dots, Quantum well lasers, Green's functions, semiconductor lasers

\footnotetext{
${ }^{1}$ Electronic mail: marco.vallone@polito.it

The research leading to these results has received funding from the European Community's Seventh Framework Programme (FP7/2007-2013) under grant agreement $\mathrm{n}^{\circ} 224338$ (FAST-DOT project).
} 


\section{INTRODUCTION}

One of the central subjects in optimizing the design of novel semiconductor laser devices based on quantum dots (QDs) is a quantitative description of various electronic scattering processes.[1] Self-assembled QDs, grown by the StranskiKrastanow method [2] consist of nanometer sized protrusions on a thin, quasi-two-dimensional wetting layer (WL). Numerous realizations consist of p-doped-intrinsic-n-doped (p-i-n) structures allowing an electrical injection; at the center of the intrinsic region, several layers of QD-WL systems are stacked together, separated by spacers $\mathrm{S}_{1}, \mathrm{~S}_{2}$ thick enough (with bulk-type density of states) to exclude vertical electronic coupling [3].

In optoelectronic applications (e.g. lasers and optical amplifiers) carriers are injected from bulk-type $\mathrm{S}_{1}, \mathrm{~S}_{2}$ spacers in the two-dimensional continuum of WL states and subsequently are captured into one of the bound, quasi 0-D states of QDs (the ground state GS and/or one of the $M$ excited states $\mathrm{ES}_{i}, i=1 \ldots M$ ). Fig. 1 shows a typical QD-based structure, much simplified: for the conduction band, electronic energy levels of QD-WL subbands GS, ES End $_{i}$ WL are respectively indicated as $\varepsilon_{G S}, \varepsilon_{E S_{i}}$ and $\varepsilon_{W L}$. A similar plot could be drawn for the holes in valence band.

In a similar way, in semiconductor lasers based on multi-quantum wells (MQWs), carriers are injected through bulk layers in the active region, where they are captured in one of the quasi-2D confined energy levels of QW, relaxing in the end on the ground state.

A major concern in this context is that each level of QW (or of QD-WL) is not an insulated system: in general, each energy level is affected by all the other QW levels in a very complicated way. Furthermore, each of them is also affected by levels and populations belonging to different QWs (or QD-WLs), the nearest neighbors in particular.

The Coulomb potential matrix, Non-Equilibrium Green's Function (NEGF) and Density Matrix (DM) formalisms provide solid conceptual basis for an investigation of this kind and will be briefly revised in Sect. 2, adapting them to the context of interest. In Sect. 3, coupling between levels in the same QW or QD-WL system will be presented as selfenergy corrections in an original way, showing how it contributes to levels renormalization. In Sec. 4, applications of the formalism are illustrated by a few examples and the same formulation is extended to QW-QW and QD-QD coupling, then in Sect. 5 conclusions will be drawn.

\section{REVIEW OF THE FORMALISM}

The NEGF formalism (also known as the Keldysh formalism) provides a microscopic theory for quantum transport including interactions. Formal descriptions of it are available in literature, $[4,5,6,7,8,9]$ but normally their formulation is based on concepts which are unfamiliar to most device physicists and probably its rigorous description may be 
omitted to render NEGF accessible and understandable enough to make use of it with benefit. In the following lines of this paragraph a brief introduction to NEGF and other useful concepts are given, keeping them as simple as possible.

In a single-particle description, if $\Psi$ is the state of energy $E$ of a particle in a system described by the Hamiltonian $H$, the non-homogeneous Schrödinger equation $(E-H) \Psi=S$ holds, where $S$ represents an excitation. The response to a delta-like excitation is defined as the Green's function $G$ associated to $H$. In an operatorial form, we may write it as $(E-H) G=I$, where $I$ is the identity matrix, so $G$ is defined in single particle formalism as $G=(E-H)^{-1}$. The solution $G\left(\vec{r}-\vec{r}^{\prime}\right)$ in the space of coordinate $\vec{r}$ corresponds both to a wave travelling outwards from (causal) and inward to (anti-causal) the point of excitation. A common way to distinguish them is to extend $G$ to the complex plane and move the pole of $G$ a bit out from the real axis of an infinitesimal real, positive quantity $\eta$, obtaining two different Green's functions: the retarded (causal) one, $G^{R 0}=(E-H+\mathrm{i} \eta)^{-1}$ and the advanced (anti-causal) one $G^{A 0}=(E-H-\mathrm{i} \eta)^{-1}$. The function $G^{R 0}$ is also called the propagator because it describes the coherent propagation of a particle from $\vec{r}$ to $\vec{r}^{\prime}$ or, in the conjugate space, a particle with energy $E$ that propagates with momentum $\vec{k}$. We observe that $G^{R 0}$ and $G^{A 0}$, expressed in the coordinate space, are complex conjugate, so in the operatorial formulation $G^{A 0}$ is the hermitian adjoint of $G^{R 0}$. As a consequence, in the followings we will refer to them respectively as $G^{0}$ and $G^{0 \dagger}$.

In a Second Quantization description, $G^{R 0}$ is defined as a particle that is destroyed at $\vec{r}^{\prime}$ at a time $t^{\prime}$ and created at $\vec{r}$ at a later time $t$, so it is described by temporal-ordered products of creation $\Psi^{\dagger}(\vec{r}, t)$ and annihilation $\Psi\left(\vec{r}^{\prime}, t^{\prime}\right)$ operators at different times. There are no real advantages in going through its full Second Quantization formulation as in the cited references, as long as we limit our description in a quasi-equilibrium context, which is exactly what we will do.

In presence of plasma, the propagation of a particle can be viewed in the context of Feynman diagrams as an infinite sum of virtual elementary scattering processes. The result is a propagator dressed with a retarded self-energy $\Sigma$, representing the effects of a propagation in a plasma that dynamically screens the interaction.[8,9] The Green's function $G$ is given as the solution of the Dyson equation $G=G^{0}+G^{0} \Sigma G$, where $G^{0}$ is the unperturbed (free) propagator. Solving for the retarded $G$, we have

$$
G(E)=\frac{1}{E+i \eta-\Sigma-H}
$$

whereas $G^{\dagger}$ is given by its hermitian conjugate.

Calculating the self-energy $\Sigma$ is a very complicated task and, for real systems like solids, approximations are usually needed. One of them is the $G W$ approximation, so called from the mathematical form $\Sigma=G W$ that the self-energy 
takes, as the product between the retarded Green's function $G$ and the dynamically screened interaction $W$. Diagrammatically the situation is represented in Fig. 2 as a particle (the dressed propagator) that interacts with the medium, emitting and reabsorbing a virtual photon/phonon (the waved line), representing the dynamically screened interaction. The "bubble" $G \otimes W$ is a retarded self-energy $\Sigma$, because it is defined by the retarded, causal propagator. The advanced self-energy is defined as its adjoint $\Sigma^{\dagger}$. Still looking at Fig. 2, the self-energy is shown to be the result of an infinite summation of diagrams. A very important concept is the spectral function

$$
\begin{aligned}
& A=i\left(G-G^{\dagger}\right)=\frac{\Gamma}{(E-H-\operatorname{Re}(\Sigma))^{2}+(\Gamma / 2)^{2}} \\
& \Gamma=-2 \operatorname{Im}(\Sigma)
\end{aligned}
$$

whose trace represents the density of states of the system, so it can be regarded as a generalized density of states. It only apparently has a Lorentzian shape, given that $\Sigma$ is in general energy-dependent. Its real part $\operatorname{Re}(\Sigma)$ has the effect to shift (renormalize) the peak of $A$ from its unperturbed position (the eigenvalue of $H$ measured in absence of plasma), whereas its imaginary part $\operatorname{Im}(\Sigma)$ gives the level a broadening $\Gamma$.

A particle in one of the QD-WL levels has not an infinite lifetime, because of the coherence loss of its motion due to scattering events. In presence of other levels, the particle can decay in a lower level (capture from WL to QD), relax toward GS, or escape from it. Looking at (1), in the denominator the unperturbed Hamiltonian appears the renormalized operator $H+\Sigma$, in which $\Sigma$ is not hermitian, generally, unlike $H$. Adding and subtracting $\Sigma^{\dagger} / 2$, we obtain $H+\Sigma=H+\operatorname{Re}(\Sigma)-i \Gamma / 2$ where $\Gamma=i\left(\Sigma-\Sigma^{\dagger}\right)$ is the same real $\Gamma$ that appears in (2), but it can be given, in a "multienergy-level" context, a different meaning: it can be viewed as the sum of two scattering functions, known in the Keldysh formalism as the in-scattering $-i \Sigma^{<}$and out-scattering $i \Sigma^{>}$rate functions (the imaginary unit and signs are a matter of convention and are related to the Keldysh formulation: its sum remains real):

$$
\Gamma(E)=i\left(\Sigma(E)-\Sigma^{\dagger}(E)\right)=-i \Sigma^{<}(E)+i \Sigma^{>}(E)
$$

in which we have indicated explicitly the energy dependence.

The inverse of the lifetime of a particle on a given level in fact is proportional to the sum of escape rates from it, up or down in energy (out-scattering) and the capture rate in it (in-scattering). This can also be viewed as the fact that an escape of an electron can also be seen as a capture of a hole and between the two processes there is a complex conjugation operation, as there is between $\Sigma$ and $\Sigma^{\dagger}$ or between $-i \Sigma^{<}$and $i \Sigma^{>}$.

To come to the point, the imaginary part of self-energy is strongly related to capture, relaxation and escape rates through (3). In order to precisely see in which way, it is necessary to introduce a further concept: the correlation functions $G^{<}$and $G^{>}$. A distribution function $f(\vec{k})$ can be thought of the diagonal elements of a more general density 
matrix $\rho\left(\vec{k}, \vec{k}^{\prime}, t\right)$, which includes additional information regarding the phase correlations between states $|\vec{k}\rangle$ and $\left|\vec{k}^{\prime}\right\rangle$ at equal time $t$. A still more general function can be defined as $G^{<}\left(\vec{k}, \vec{k}^{\prime} ; t, t^{\prime}\right)$ correlating the amplitude of a state $|\vec{k}\rangle$ at time $t$ with a state $\left|\vec{k}^{\prime}\right\rangle$ at a different time $t^{\prime}$. Its diagonal elements $G^{<}(\vec{k} ; t)$, Fourier transformed respect to $t$ to give $G^{<}(\vec{k} ; E)$ and integrated in energy $E$, constitute the particle distribution $f(\vec{k})$. Instead, just as a matter of definition, if we Fourier transform $G^{<}(\vec{k} ; E)$ respect to $\vec{k}$ and integrate in energy, we get the electron density $n(\vec{r})$ in ordinary space. It can be easily verified that, at thermodynamic quasi-equilibrium, when all the states are occupied according to a Fermi function $f_{0}(E)$, the electron density can also be evaluated as the integral of $f_{0}(E) A(\vec{k}, E)$ in energy and momentum. It can be demonstrated [10] that at quasi-equilibrium $f_{0}(E) A(\vec{k}, E)$ and $G^{<}(\vec{k} ; E)$ coincide and that the corresponding holes correlation function $G^{>}(\vec{k} ; E)$ at quasi-equilibrium coincides with $\left(1-f_{0}(E)\right) A(\vec{k}, E)$.

Moreover, it can be demonstrated $[11,12]$ that the relation between the scattering functions and the broadening of the spectral function is, at quasi-equilibrium:

$-i \Sigma^{<}=f_{0}(E) \Gamma(E) ; \quad i \Sigma^{>}=\left(1-f_{0}(E)\right) \Gamma(E)$

Furthermore, the transition rate in or out of a level is given by one of the following equivalent expressions:

$$
\tau^{-1}=\frac{\Gamma}{\hbar}=\frac{-i\left(\Sigma^{<}-\Sigma^{>}\right)}{\hbar}=\frac{i\left(\Sigma-\Sigma^{\dagger}\right)}{\hbar}
$$

that, at quasi-equilibrium reduce to:

$\tau_{e q}^{-1}=-\frac{2}{\hbar} \operatorname{Im}(\Sigma)$

and in the retarded self-energy $\Sigma$ we have all the information we need.

\section{LEVELS COUPLING AND SELF-ENERGIES}

First question is: how do we calculate QD, WL and QW levels? They are the eigenvalues of the unperturbed Hamiltonian $H_{0}=-\frac{\hbar^{2}}{2 m} \nabla^{2}+U(z)$, where $U(z)$ is the potential profile for the considered band (e.g. the conduction band). We call them unperturbed levels (or subbands). 
Let us consider a subband as if it were insulated, occupied by a single particle. The wave function $\Psi_{n}$ associated to it (where $n$ represents the ensemble of its quantum numbers) obeys the Schrödinger equation $H_{0}\left|\Psi_{n}\right\rangle=E\left|\Psi_{n}\right\rangle$ and we define the associated, retarded Green's function matrix $G^{0}$ as the solution of the equation $\left(E+i \eta-H_{0}\right) G^{0}(E)=I$. If two levels are coupled, a term describing their interaction energy must be present in the Hamiltonian. Let $\Psi_{m}(\vec{r})$ and $\Psi_{n}\left(\vec{r}^{\prime}\right)$ be states in subbands $m$ and $n$. The interaction (coupling) between them can be viewed as an exchange of a virtual photon, that is a scattering event with exchange of momentum $\vec{q}$ and frequency $\omega$. If $\Psi_{i}$, and $\Psi_{j}$ are the states after scattering, the interaction Hamiltonian element is given by:

$$
\begin{aligned}
V_{i j ; n m}=\int d \vec{r} d \vec{r}^{\prime} \Psi_{n}^{*}\left(\vec{r}^{\prime}\right) \Psi_{m}(\vec{r}) \\
\cdot V_{s}\left(\left|\vec{r}-\vec{r}^{\prime}\right|\right) \Psi_{i}^{*}(\vec{r}) \Psi_{j}\left(\vec{r}^{\prime}\right)
\end{aligned}
$$

in which $V_{s}$ is the Coulomb interaction matrix potential, dynamically screened by carriers plasma. This potential matrix expression is very general and can describe in principle all scattering events. If we are dealing with coupling effects only, there is no change of band and we have $i=m, j=n$, but for the moment we maintain the general expression. In the hypothesis that QW, WL and QD wavefunctions $\Psi_{n}(\vec{r})$ can be factorized as $[1,13]$ :

$\Psi_{n}(\vec{r})=\varphi_{l_{n}}(\vec{\rho}) \zeta_{\sigma_{n}}(z) u_{n}(\vec{r})$

where $\varphi_{l_{n}}(\vec{\rho})$ is the in-plane component, $\zeta_{\sigma_{n}}(z)$ the perpendicular $z$-component and $u_{n}(\vec{r})$ the Bloch function, a great simplification can take place and we assume this hypothesis throughout (here $l$ and $\sigma$ are the in-plane and perpendicular wave function quantum number, that combine in $n$ at the end of calculation). In this hypothesis, operating a Fourier transform of the $z$-component of $\vec{q}$, the interaction Hamiltonian element becomes:

$V_{i j ; n m}(\vec{q}, \omega)=\frac{2 \pi e^{2}}{q} \frac{F_{i j ; n m}(q) P_{i j ; n m}(q)}{\varepsilon(q, \omega)}$

where

$$
F_{i j ; n m}(q)=\int d z d z^{\prime} \zeta_{\sigma_{n}}^{*}\left(z^{\prime}\right) \zeta_{\sigma_{m}}(z) \exp \left(-q\left|z-z^{\prime}\right|\right) \zeta_{\sigma_{i}}^{*}(z) \zeta_{\sigma_{j}}\left(z^{\prime}\right)
$$

and

$$
P_{i j ; n m}(q)=\int d^{2} \vec{\rho} \varphi_{l_{i}}^{*}(\vec{\rho}) \varphi_{l_{m}}(\vec{\rho}) \exp (-i \vec{q} \cdot \vec{\rho}) \int d^{2} \vec{\rho}^{\prime} \varphi_{l_{j}}\left(\vec{\rho}^{\prime}\right) \varphi_{l_{n}}^{*}\left(\vec{\rho}^{\prime}\right) \exp \left(i \vec{q} \cdot \vec{\rho}^{\prime}\right)
$$

Here the dielectric constant $\varepsilon(q, \omega)$ may have the form given, for example, in Ref.(8) or in our previous work (see Ref. (15), Eq.(6)), in which a screened electron-electron and electron-phonon coupled interaction were considered. About wavefunctions, various levels of approximation can be followed and the exact calculations of coupling factors $F_{i j ; n m}$ 
and $P_{i j ; n m}$ is out of the scope of the present work. What is here important to notice is the fact that (8) can represent not only the interaction potential matrix in any scattering event, but also the coupling energy between subbands.

If there are several subbands in the conduction band, a given state of the system $\Psi$ is represented by the vector $|\Psi\rangle=\left|\Psi_{S_{1}}, \Psi_{W L}, \Psi_{G S}, \Psi_{E S_{1}}, \ldots, \Psi_{E S_{M}}, . \Psi_{S_{2}}\right\rangle$. If all subbands were uncoupled (that is, if a particle in a subband is not affected by other subbands), the single particle Hamiltonian of the system would be diagonal in the $\left\{\Psi_{i}\right\}$ basis and its eigenvalues would be just the levels $\varepsilon_{i}$, where $i=\mathrm{S}_{1}, \mathrm{WL}, \mathrm{ES}_{1}, \ldots$, etc., calculated solving the Schrödinger equation $H_{0}\left|\Psi_{n}\right\rangle=E\left|\Psi_{n}\right\rangle$. If carriers injection is considered, following the many-particle propagator formalism, but keeping subbands uncoupled, the Hamiltonian is still diagonal, but its eigenvalues are modified to $\varepsilon_{n}+\Sigma_{n}$, where $\Sigma_{n}$ is the diagonal self-energy contribution, that in the $G W$ formalism assumes the form:

$\Sigma_{n}(\vec{k})=-\frac{1}{\beta} \sum_{q, \omega} V_{n n ; n n}(\vec{q}, \omega) G_{n n}(\vec{k}-\vec{q}, \omega)$

Here $\beta$ is the inverse temperature in energy units and $G_{n n}$ is the particle propagator for the $n$-th subband. $\Sigma_{n}$ originates the usual exchange and Coulomb-hole contributions and can be evaluated at the desired level of approximation. $[9,10,14]$

This is just the hypothesis normally accepted in many scattering calculations, justified by the fact that off-diagonal elements are small compared with the energy separation between subbands.[15,16] The possibility to accept this simplification depends on the cases on study and on the purposes of calculation. The model we present here allows to give quite a simple expression of the problem, keeping at least the greater part of the correction coming from coupling effects.

When subband coupling is considered, we assume that each subband is coupled only to its nearest neighbors in energy, with an interaction potential $V_{m n ; n m}$. In this way, disregarding spacers $\mathrm{S}_{1}, \mathrm{~S}_{2}$ contributions, the Schrödinger equation $H|\Psi\rangle=E|\Psi\rangle$ assumes a tri-diagonal matrix form in which off-diagonal elements between columns $m$ and $n$ are given by $V_{m n ; n m}$, whereas on-diagonal elements are the usual kinetic and potential terms $H_{0}+V_{n n, n n}$. Still following the propagator formalism, the off-diagonal elements give origin to off-diagonal self-energy terms $\Sigma_{m n}$, still having the form (11a), with $V_{m n ; n m}, m \neq n$,instead of $V_{n n ; n n}$.

$$
\Sigma_{m n}(\vec{k})=-\frac{1}{\beta} \sum_{q, \omega} V_{m n ; n m}(\vec{q}, \omega) G_{n n}(\vec{k}-\vec{q}, \omega)
$$


If $H$ is the Hamiltonian, we define the associated Green's function matrix as the solution of the equation $(E-H) G(E)=I$, where $E-H$ is tri-diagonal too:

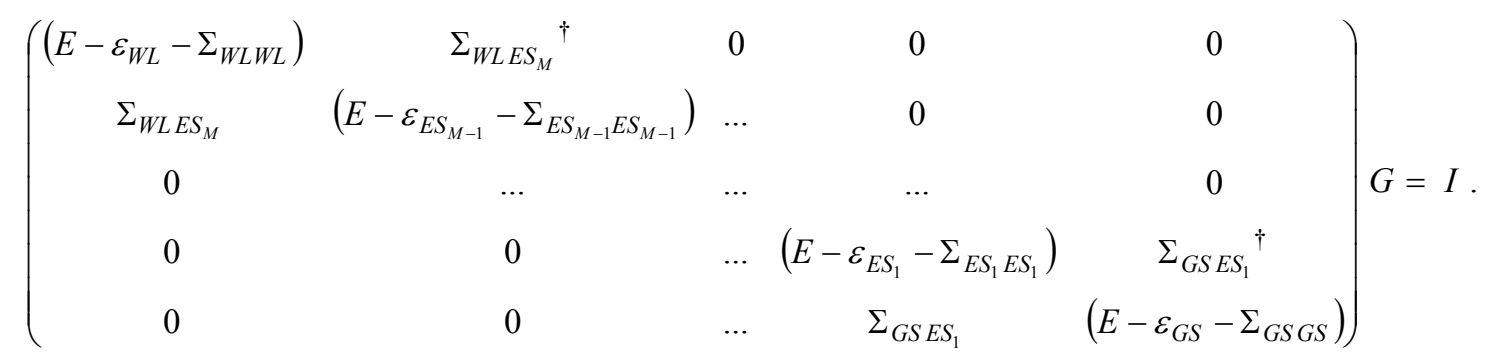

Each diagonal element of the retarded function $G^{R}$ associated with $G$ represents the response of any subsystem (GS, $\left.\mathrm{WL}, \mathrm{ES}_{\mathrm{i}}, \ldots ..\right)$ due to an excitation at any other. For example, solving for the ground state $G_{G S}^{R}$, in the basis of $H$ eigenstates, we find the following expression, having considered only two QD excited states, as an example:

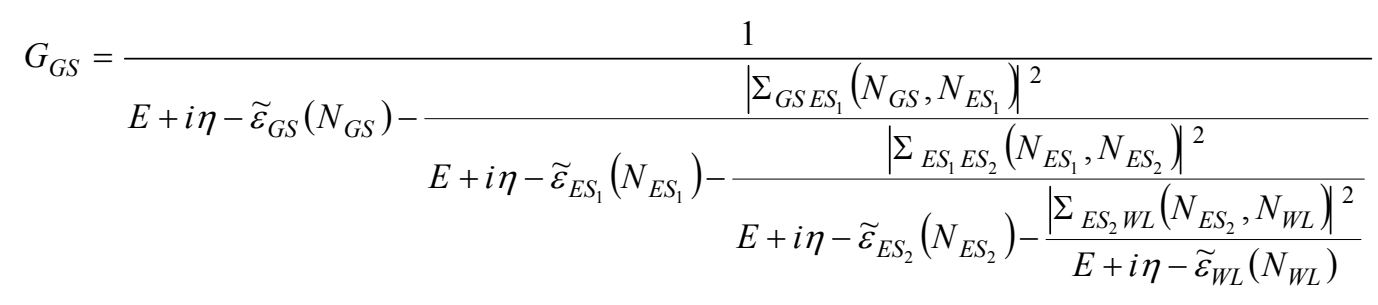

Here we have indicated as $\widetilde{\varepsilon}_{i}$ the eigenvalues $\varepsilon_{i}$, corrected for the diagonal elements of self-energy $\Sigma_{i i}\left(N_{i}\right)$ due to its level population effects only, whose carrier density is $N_{i}$, that is $\widetilde{\varepsilon}_{i}=\varepsilon_{i}+\Sigma_{i i}\left(N_{i}\right)$. For brevity of notation, we have not indicated the weak energy-dependence of self-energy on $E$, understood throughout.

It is worthy of note the fact that the denominator of (13) has a continued fractions expression and in particular it takes the form of the energy expectation value of a Brillouin-Wigner perturbation expansion:[17]

$$
E=\widetilde{\varepsilon}_{0}-\frac{\left|\Sigma_{01}\right|^{2}}{E-\widetilde{\varepsilon}_{1}-\frac{\left|\Sigma_{12}\right|^{2}}{E-\widetilde{\varepsilon}_{2}-\ldots}} .
$$

This expression is more complicated compared with the more usual Rayleigh-Schrödinger series of conventional perturbation theory, due to the fact that the energy $E$ appears in all denominators.

The Hamiltonian eigenvalue for the GS subband is given by the poles of (13), that is, the roots of the equation:

$$
E-\varepsilon_{G S}-\Sigma_{G S G S}\left(E, N_{G S}\right)-\Sigma_{G S, o f f}\left(E, N_{G S}, N_{\text {others }}\right)=0
$$


where $\Sigma_{G S G S}\left(N_{G S}\right)$ is the self-energy diagonal term that depends on GS carrier density $N_{G S}$ only, to be evaluated e.g. as in Ref. (9), Ref. (10) and Ref. (13). $\Sigma_{G S, o f f}$ is the off-diagonal self-energy correction, due to coupling of GS with all other subbands, and depends, in a complicated by straightforward way, on carrier densities on each level, as shown by (13), here summarized as $N_{\text {others }}$. Each subband has its own correction, due to the presence of all higher levels. Equations similar to (13)-(15) hold for each level $\mathrm{ES}_{\mathrm{i}}$ and for WL, allowing to evaluate all Hamiltonian eigenvalues, corrected for off-diagonal contributions, in the hypothesis done.

The role of the off-diagonal contribution to self-energy consists in an extra-renormalization of GS toward low energies (so, further reducing the energy-gap), due to a repulsion effect between subbands, as indicated in Sect. IV. This formulation is general; it does not depend on what self-energy formulation is used and applies both to QD and QW.

\section{APPLICATIONS AND EXTENSIONS OF THE FORMALISM}

\section{A) Coupling among different levels in the same $Q W$ or $Q D$.}

As a first example of application, we consider a $120 \AA / 120 \AA$ unstrained InGaAsP quantum well / barrier system, emitting at $1550 \mathrm{~nm}$, with a conduction band offset of $177 \mathrm{meV}$ between the well and the barrier materials. We calculated the position of conduction band levels in effective mass approximation, finding two bound levels, whose confinement energies are $148 \mathrm{meV}$ (GS) and $66 \mathrm{meV}$ (ES), quite close each other due to the wide QW. The explicit expression of off-diagonal self-energy contribution $\Sigma_{G S, \text { off }}$ have a simple expression, especially expressing the dielectric function $\varepsilon(q, \omega)$ in the static limit of the RPA Single Plasmon Pole. In this case, in (11) the propagator is given by (see [8])

$$
G_{G S}\left(\vec{k}-\vec{q}, \omega_{r}\right)=\frac{n_{G S}\left(|\vec{k}|, N_{G S}\right)}{\mathrm{i} \hbar p_{G S}+\mathrm{i} \hbar \omega_{r}+\hbar^{2}|\vec{k}-\vec{q}|^{2} /\left(2 m_{e f f}\right)+\varepsilon_{E S}}
$$

where $\hbar$ is the reduced Planck's constant, $n_{G S}\left(k, N_{G S}\right)$ is the Fermi distribution for the GS in-plane $\vec{k}$ state. Here $\varepsilon_{E S}$ is the unperturbed energy of the ES-subband, $m_{\text {eff }}$ is the carrier effective mass and $p_{G S}, \omega_{r}$ are respectively the frequency of the GS state and the exchanged quantum of energy during the interaction, which have to be treated according to the Matsubara formalism.[8,9,14] Frequency $\omega_{r}$ summation yields the occupation factor $-n_{E S}\left(|\vec{k}-\vec{q}|, N_{E S}\right)$ of the ES state. Integrating in the QW plane and multiplying by two to consider the spin 
summation, we find the expression (17), in which, for the translation invariance, $P_{E S G S ; G S E S}$ is unity, whereas $F_{E S G S ; G S E S}$ is readily evaluated from the wave functions expressions:

$\Sigma_{G S E S}\left(|\vec{k}|, N_{G S}, N_{E S}\right)=\frac{2 e^{2}}{\beta \varepsilon_{S}} \int_{0}^{\infty} n_{G S} n_{E S} \frac{F_{E S G S ; G S E S}(q)}{q+\kappa\left(N_{G S, E S}\right)} q d q$,

having indicated with $n_{G S}, n_{E S}$ respectively the occupation factors $n_{G S}\left(|\vec{k}|, N_{G S}\right)$ and $n_{E S}\left(|\vec{k}-\vec{q}|, N_{E S}\right)$, for brevity of notation. Here $\kappa\left(N_{G S, E S}\right)=\left[\kappa\left(N_{G S}\right)^{-1}+\kappa\left(N_{E S}\right)^{-1}\right]^{-1}$ is the density dependent GS-ES reduced screening wave vector. The integral in $q$ converges quickly, because of the form-factor $F_{E S G S ; G S E S}(q)$ that drops rapidly in $q$, and can be numerically evaluated without any problem. This expression is purely real and is suitable only to evaluate renormalization. In order to evaluate transition rates, a full dynamic expression of dielectric function should be considered and will be addressed in a future Paper. The position of GS and ES results, from (13-15):

$\breve{\varepsilon}_{G S}=\frac{\varepsilon_{G S}+\varepsilon_{E S}+\Sigma_{G S}+\Sigma_{E S}}{2}-\frac{\Delta \varepsilon_{E S-G S}}{2} \sqrt{4\left|\frac{\Sigma_{G S E S}}{\Delta \varepsilon_{E S-G S}}\right|^{2}+1}$
$\breve{\varepsilon}_{E S}=\frac{\varepsilon_{G S}+\varepsilon_{E S}+\Sigma_{G S}+\Sigma_{E S}}{2}+\frac{\Delta \varepsilon_{E S-G S}}{2} \sqrt{4\left|\frac{\Sigma_{G S E S}}{\Delta \varepsilon_{E S-G S}}\right|^{2}+1}$

where $\Delta \varepsilon_{E S-G S}=\varepsilon_{E S}+\Sigma_{E S}\left(N_{E S}\right)-\left[\varepsilon_{G S}+\Sigma_{G S}\left(N_{G S}\right)\right]$ is the difference between diagonally-renormalized energy levels.

In Fig. 3 levels position is reported, considering thermalized electrons with $\vec{k}=0$, for several values of GS carrier density. $\mathrm{ES}_{1}$ carrier density has been evaluated at thermal quasi-equilibrium and diagonal parts of self-energy have been calculated in the static limit of RPA. When $\mathrm{N}_{\mathrm{GS}}$ starts increasing, both levels reduce their energy: this is the usual energy renormalization. When $N_{G S}$ exceeds $2 \cdot 10^{12} \mathrm{~cm}^{-2}$, the population of ES starts affecting GS, and vice versa: we have a further renormalization of GS and a reduction of renormalization for ES and at very high density the effect is quite considerable, due to the closeness of the levels.

A similar calculation applies to QDs, with a more complicated treatment of $P_{E S G S ; G S E S}$ and $F_{E S G S ; G S E S}$ and will be omitted here for brevity. From a qualitative point of view, carriers in QDs are normally too few to considerably shift the levels, but WL can be heavily populated by current injection. The correct way to keep into account energy renormalization in QD is to make use of an equation like (18). Discarding the very low correction due to ES, the GS energy level, as a function of carrier density in GS and in WL, assumes the form: 


$$
\begin{aligned}
\widetilde{\varepsilon}_{G S}= & \frac{\varepsilon_{G S}+\varepsilon_{W L}+\Sigma_{G S}\left(N_{G S}\right)+\Sigma_{W L}\left(N_{W L}\right)}{2} \\
& -\frac{\Delta \varepsilon_{W L-G S}}{2} \sqrt{4\left|\frac{\Sigma_{G S W L}\left(N_{G S}, N_{W L}\right)}{\Delta \varepsilon_{W L-G S}}\right|^{2}+1}
\end{aligned}
$$

where $\Delta \varepsilon_{W L-G S}=\varepsilon_{W L}+\Sigma_{W L}\left(N_{W L}\right)-\left[\varepsilon_{G S}+\Sigma_{G S}\left(N_{G S}\right)\right]$. The interaction self-energy $\Sigma_{G S W L}\left(N_{G S}, N_{W L}\right)$ has still the form (17) (in which the subscript WL substitutes ES) with an opportune calculation of $F_{W L G S ; G S W L}$ integral according to (9).

Other situations can occur, for example, not limiting interactions between the nearest levels. In general, a little coupling does exist among all levels, but in this case the task to keep into account all corrections is very challenging and can be addressed only numerically, not being possible to obtain a conceptually simple formula as the continued-fraction one. For a treatment of the general coupling, although presented not in an explicit way, see e.g. Ref. (16).

\section{B) Coupling among levels of different QDs or QWs}

It is well known that, in narrowly-spaced and shallow quantum wells, coupling energy between levels can split them, originating a miniband (superlattices). In a similar way, this can also happen in stacked self-assembled and narrowly spaced InAs/GaAs QD layers, with the complication that QDs are generally of different size, giving origin to different energy position of QD levels. Let us consider two stacked, narrowly spaced QD of different size, as in Fig. 4(a). Their levels are at different energies and coupling is inefficient. Applying electric field oriented along the stacking axis via a voltage applied between the back n-contact and the top p-contact, we can tune the coupling making their levels coincide, couple and split, as in Fig. 4(b). The off-diagonal self-energy formalism presented in the previous Section can be efficiently exploited to describe this phenomenology, too. As an example, for the situation depicted in Fig. 4(a), we indicate with $\breve{\varepsilon}_{G S}\left(N_{G S}, N_{\text {others }}\right)$ the renormalized energy level of $\mathrm{GS}_{1,2}$ given by the root of (15) (and similarly $\breve{\varepsilon}_{E S_{n}}$ for $\mathrm{ES}_{\mathrm{n}}$ ). When we tilt the bands applying an electric field, we can reach a situation in which - for example - the ground states of the two QD coincide, in absence of coupling, so they both have the same energy $\breve{\varepsilon}_{G S}$. The level splitting can be described writing a matrix equation like $(E+i \eta-H) G^{R}(E)=I$, as usual:

$\left(\begin{array}{cc}G_{G S} & G_{c} \\ G_{c} & G_{G S}\end{array}\right)\left(\begin{array}{cc}E+i \eta-H_{Q D} & \Sigma_{G S_{1}, G S_{2}} \\ \Sigma_{G S_{1}, G S_{2}}^{\dagger} & E+i \eta-H_{Q D}\end{array}\right)=\left(\begin{array}{ll}1 & 0 \\ 0 & 1\end{array}\right)$

in which $H_{Q D}$ is the Hamiltonians of the QDs considered insulated and $\Sigma_{G S_{1}, G S_{2}}$ is the inter-dot coupling Hamiltonian for GS. Solving for $G_{G S}$, we find, in the basis of $H_{Q D}$ eigenstates: 


$$
G_{G S}=\frac{1}{E+i \eta-\breve{\varepsilon}_{G S}-\frac{\left|\Sigma_{G S_{1}, G S_{2}}\right|^{2}}{E+i \eta-\breve{\varepsilon}_{G S}}}
$$

The eigenvalues of the total Hamiltonian, i.e. the GS energy, recalculated keeping into account the inter-dot coupling, are given by the poles of (21), that is:

$$
\varepsilon_{G S_{-} \text {with coupling }}=\breve{\varepsilon}_{G S}\left(N_{G S}, N_{\text {others }}\right) \pm \Sigma_{G S_{1}, G S_{2}}\left(N_{G S_{1}}, N_{G S_{2}}\right) \text {. }
$$

As expected, the energy level is split by the effect of coupling in a singlet and a triplet state.

Assuming a QD confining potential as in Ref. (18), $\Sigma_{G S_{1}, G S_{2}}$ can be readily evaluated; for example, if $\breve{\varepsilon}_{G S}=-80 \mathrm{meV}$ respect to the WL for both QDs and electron mass is 0.060 free electron mass, the GS levels energy for the QD couple becomes as in Fig. 5: we can see that for QD separated less than $10 \mathrm{~nm}$ the splitting is quite considerable.

This effect is well known, but with the present formalism it is straightforward to account both for the Coulomb potential screening effect and the Fermi band-filling, as done in (17), assigning $N_{G S_{1}}, N_{G S_{2}}, N_{\text {others }}$ to self-energy components in the opportune way, as done for example building (17) - (19).

These concepts applies to QW of different width as well, in which an electric field applied along the growth direction can be used to tune level positions: when they coincides, they split according to (22), in which $\Sigma_{G S_{1}, G S_{2}}$ is now the inter-QW coupling energy for the two QW ground-states.

\section{CONCLUSIONS}

If we consider a single QW or QD, the calculation of levels energy, given by poles of equations similar to (13), presents the advantage of allowing the renormalization of each level for carriers injection separately in the standard way, delegating to off-diagonal potentials matrix terms $V_{i j}$ all the difficulty to keep into account levels coupling. Although the physics of level coupling is well known, this is not true for the applications it can explore. The coupling here depicted for electrons in coupled QDs can be obtained both for electrons and holes, giving the opportunity to explore many novel physical concepts, as voltage tunable magnetic properties and manipulation of the hole spin in quantum computing. The presented Green's function based approach gives a more intuitive view of the various meanings and roles that a concept like "self-energy" can have. The concept of coupling is viewed linked to a general potential matrix 
and can be given the same form for all kinds of coupling, in which off-diagonal self-energy contributions assume each time a different conceptual meaning. Furthermore, population effects belonging to each level arise naturally without any confusion, as arguments of the related self-energy components.

We point out that our purpose is not to give a full and self-consistent calculation of coupling contribution to level position in energy. It is well evident that considering coupling effects beyond nearest-neighbors (e.g. between GS and $\mathrm{ES}_{3}$ ) resulted in a more accurate calculation, but this can obtained only considering a full squared Hamiltonian, instead of a tri-diagonal one in (12). This is surely possible to be implemented - it is a matter of software -, but it prevents the comprehension of the underlying physics and the building of explicit expressions like (19), (21) and (22).

More, we stress that the same formalism apply unchanged also to hole levels: in this case, the corrections are also more necessary given that levels are less spaced each other. In principle, nothing changes in the formalism, if electrons-holes interaction is considered, too. If we only consider interactions between levels having the same principal quantumnumber (e.g. $e_{0}-h_{0}$, or $e_{1}-h_{1}$, but not also $e_{0}-h_{1}$ ) that normally result in the greatest contribution, it is sufficient to insert in (12) an extra row and column for each electron-hole states to couple, keeping the matrix form in (12) still tri-diagonal and the whole treatment unchanged in form and meaning. On the contrary, if all states have to be considered coupled, of course the matrix results no more tri-diagonal. The major difficulty in treatment could not be justified, if selection rules and the obtained values for $F_{i j ; n m}(q)$ and $P_{i j ; n m}(q)$ coefficients resulted very little; from time to time, the necessity to extend the description till this grade of complication should be evaluated, depending on the structure we are dealing with and the purpose of the calculation (approximate device design or basic research) we are carrying on. 


\section{FIGURE 1}

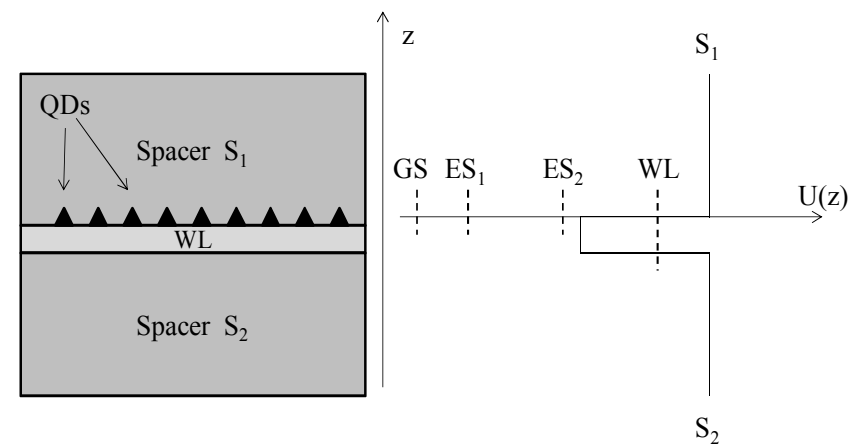

Fig. 1. On the left, a sketch of a WL-QD layer grown by Stranski-Krastanow method is shown. On the right, conduction band is shown with the confining potential U(z), the WL bound level (substantially a very thin quantum well), ground state (GS) and excited states (ES) of quantum dot. 
FIGURE 2
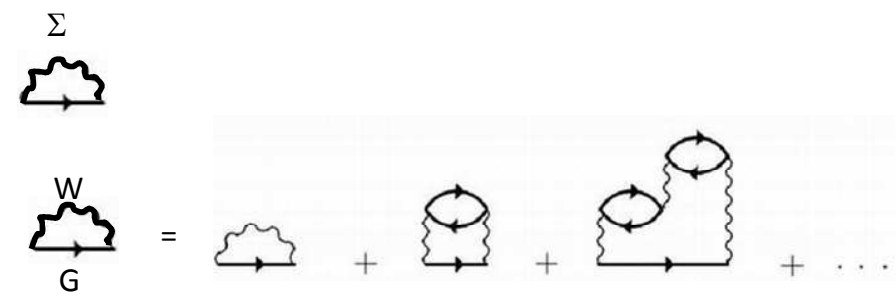

Fig. 2. The self-energy in a generally accepted approximation is represented by a propagator G multiplied by the interaction $\mathrm{W}$ and integrated over internal exchanged momentum. The effect of potential screening can be visualized as a summation over infinite ladder contributions. 


\section{FIGURE 3}
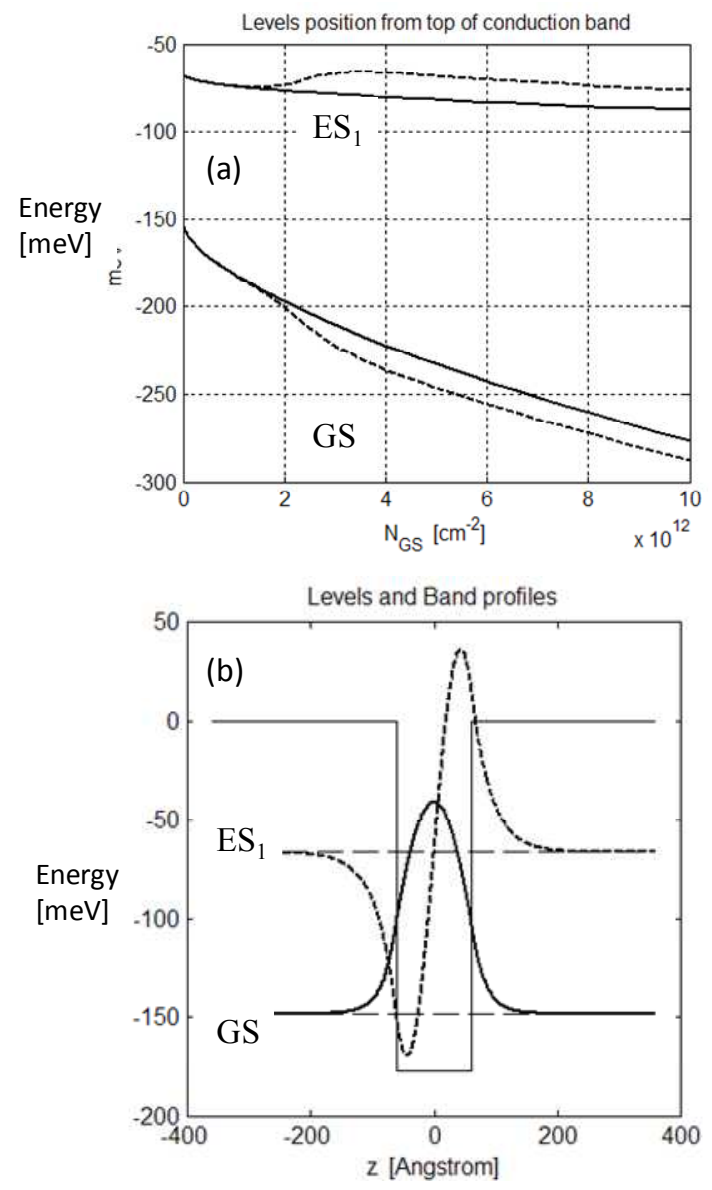

Fig. 3.

(a) Energy levels position for GS and $\mathrm{ES}_{1}$, with off-diagonal self-energy contribution (dashed lines) and disregarding them (solid), as a function of the GS two-dimensional carrier density.

(b) Well/barrier potential profile, with unperturbed (0-density) levels position, GS (solid) and ES (dashed) eigenfunctions in the $z$-direction. 


\section{FIGURE 4}

(a)

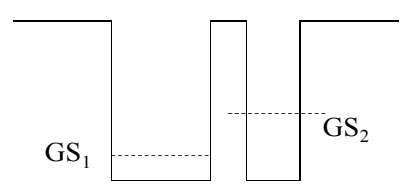

(b)

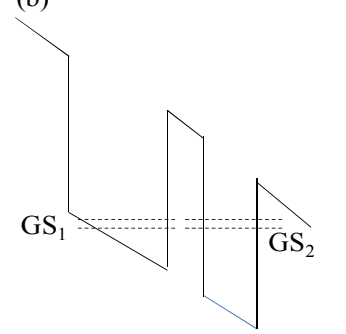

Fig. 4.

(a) Energy levels position in conduction band, for GS of two stacked QD (or two adjacent QW as well), with flat bands in absence of electric field (build-in potential is not considered here, for simplicity).

(b) Electric field can be used to fine tune the levels position. If spacers between QDs layers (or barriers between QWs) are thin enough, levels are coupled and split. 


\section{FIGURE 5}

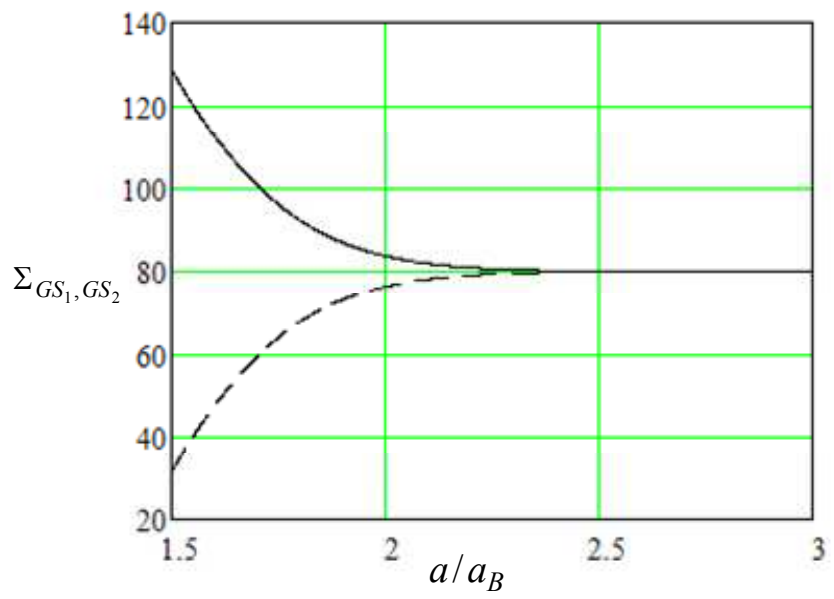

Fig.5. GS energy level splitting by effect of QD-QD coupling, for two stacked layers of self-assembled InAs/GaAs quantum dots, at null carrier density. Electron mass $m$ has been set to 0.060 free electron mass. In abscissas, $a / a_{B}$ is the layers separation in unit of Bohr radius $\hbar / \sqrt{m \breve{\varepsilon}_{G S}}$ (here $\approx 4 \mathrm{~nm}$ ). 


\section{References}

1 T. Nielsen, P. Gartner and F. Jahnke, Phys. Rev. B, 69, 23 (2004).

2 D. Bimberg, M. Grundmann, and N. N. Ledentsov, “Quantum Dot Heterostructures “ (Wiley, New York, 1999).

3 P. Borri, S. Schneider, W. Langbein and D. Bimberg,, J. Opt. A, Pure and Appl. Opt., 8 (2006).

4 P.C. Martin and J. Schwinger, Phys. Rev. 115, 6 (1959).

5 L.P. Kadanoff and G. Baym, "Quantum statistical mechanics” (Benjamin, New York, 1962).

6 L.V. Keldysh, Sov. Phys. JETP, 20 (1965).

7 H. Haug and A.-P. Jauho, "Quantum kinetics in transport and optics of semiconductors" (Springer, Berlin, 1996).

8 G.D. Mahan, "Many particle physics" (Plenum, New York, 1981).

9 A.L. Fetter and J.D. Walecka, “Quantum theory of many particle systems” (Dover Publ., Mineola (N.Y.), 2003).

10 H. Haug and S.W. Koch, Phys. Rev. A, 39, 4 (1989).

11 S. Datta, "Electronic transport in mesoscopic systems" (Cambridge Univ. Press, Ccambridge, 1995).

12 H. Haug, "Optical Nonlinearities and Instabilities in Semiconductors", (Academic Press, San Diego 1988).

13 H. Haug and S.W. Koch, "Quantum theory of the optical and electronic properties of semiconductors" (World Scientific, Singapore, 1990).

14 H. Haug and S. Schmitt-Rink, J. Opt. Soc. Am. B, 2,7 (1985).

15 M. Vallone, J. Appl. Phys., 107, 5 (2010).

16 P. Sotirelis, P. von Allmen and K. Hess, Phys. Rev. B, 47, 19 (1993).

17 J.M. Ziman, “Elements of advanced quantum theory” (Cambridge Univ. Press, Cambridge, 1969).

18 G. Burkard, D. Loss, and D.P. DiVincenzo, Phys. Rev. B, 59, 3 (1999). 